\title{
CORRELATION OF NECK CIRCUMFERENCE AND DYSLIPIDAEMIA
}

\author{
Dheeraj Kumar Reddy', Prakruthi Jaladhar ${ }^{2}$, Sudesh Shetty 3
}

1 Postgraduate Student, Department of General Medicine, Yenepoya Medical College, Mangalore, Karnataka.

${ }^{2}$ Assistant Professor, Department of General Medicine, Yenepoya Medical College, Mangalore, Karnataka.

${ }^{3}$ Associate Professor, Department of General Medicine, Yenepoya Medical College, Mangalore, Karnataka.

\section{BACKGROUND}

ABSTRACT

Anthropometric measures have been used for screening patients for cardiovascular abnormalities and metabolic syndrome since many years. The measurements generally used are height, weight, waist and hip circumference and their ratio and neck circumference. However, it might not be acceptable for all cultures to agree for such screening. Neck circumference could be an acceptable option for such patients, as it is considered as an index of upper body obesity and correlates positively with changes in blood pressure and other components of metabolic syndrome.

\section{MATERIALS AND METHODS}

The study was conducted on patients attending the outpatient department (OPD) for general health check-up at Yenepoya Medical College Hospital among 201 participants above 20 years of age and excluding cases with thyroid disorders, any neck surgery and pregnant women. Along with fasting lipid profile, neck circumference was measured in all the participants and parameters evaluated.

\section{RESULTS}

On analysis, we found a significant positive correlation between Neck circumference and Cholesterol in males and females with $\mathrm{p}<0.001$ in males and $\mathrm{p}=0.02$ in females and significant positive correlation between Neck circumference and LDL in males with $\mathrm{p}<0.001$.

\section{CONCLUSION}

Studies on similar lines are not available on Indian population. Neck circumference may be used as a simple and time saving screening measure to identify cardiometabolic risk factors like dyslipidaemia in patients.

\section{KEYWORDS}

Neck Circumference, Dyslipidaemia, Metabolic Syndrome, Obesity.

HOW TO CITE THIS ARTICLE: Reddy DK, Jaladhar P, Shetty S. Correlation of neck circumference and dyslipidaemia. J. Evolution Med. Dent. Sci. 2018;7(12):1503-1506, DOI: $10.14260 /$ jemds/2018/340

\section{BACKGROUND}

Obesity is a state of excess adipose tissue mass. Although, not a direct measure of adiposity, the most widely used method to gauge obesity is the body mass index (BMI), which is equal to Weight/Height ${ }^{2}$ (in $\mathrm{kg} / \mathrm{m}^{2}$ ). There are numerous methods to assessing overweight and obesity such as measurements of weight, height, waist circumference, hip circumference and calculation of waist-hip ratio and BMI. Due to cultural inhibitions measurement of hip, thigh or waist circumference is cumbersome in females.

US Preventive Service Task Force recommends that all adults must be screened for obesity to prevent morbidity and mortality. Hence, screening must be simple, least cumbersome, non-invasive and easily feasible like measurement of neck circumference. Upper body subcutaneous fat as estimated by Neck circumference confer risk above and beyond visceral abdominal fat.(1) Various studies indicated that neck circumference may be

'Financial or Other Competing Interest': None.

Submission 31-01-2018, Peer Review 01-03-2018,

Acceptance 08-03-2018, Published 19-03-2018.

Corresponding Author:

Dr. Prakruthi Jaladhar,

No. 1907, A Wing, Siliconia Apartment,

Kuttar, University Road,

Mangalore-575018.

E-mail: prakruthi30@gmail.com

DOI: $10.14260 /$ jemds $/ 2018 / 340$ an independent correlate of metabolic risk factors beyond BMI and waist circumference.(2) Neck circumference is considered an index of upper body obesity and correlate positively with changes in systolic and diastolic blood pressure and other components of metabolic syndrome.(3)

A global transition in disease pattern has been observed where the relative impact of infectious diseases is decreasing, while chronic diseases like cardiovascular diseases and diabetes are increasing.(4) Epidemiologists in India and International agencies such as World Health Organisation (WHO) have been sounding an alarm on rapidly rising burden of CVD for the past 15 years. It is estimated that by 2020, CVD will be the largest cause of disability and death in India with 2.6 million Indians predicted to die due to CVD. The risk of cardiovascular diseases accompanying the metabolic syndrome is approximately doubled compared with absence of the syndrome. The metabolic syndrome consists of a constellation of metabolic abnormalities that confer increased risk of cardiovascular diseases and diabetes mellitus.(5)

The major features of metabolic syndrome include central obesity, hypertriglyceridaemia, low high-density lipoprotein, hyperglycaemia and hypertension. The metabolic syndrome (MetS) is a major and escalating public health and clinical challenge worldwide in the wake of urbanisation, surplus energy intake, increasing obesity and sedentary life habits. MetS confers a 5-fold increase in the risk of type 2 diabetes mellitus (T2DM) and 2-fold the risk of developing 
cardiovascular disease (CVD) over the next 5 to 10 years. Further, patients with the MetS are at 2- to 4-fold increased risk of stroke, a 3- to 4-fold increased risk of myocardial infarction (MI) and 2-fold the risk of dying from such an event compared with those without the syndrome regardless of a previous history of cardiovascular events.(6)

The metabolic syndrome is a clustering of hyperglycaemia/ insulin resistance, obesity and dyslipidaemia.(7),(8) It is important for several reasons.

1. First, it identifies patients who are at high risk of developing atherosclerotic CVD and type 2 diabetes (T2D).(9)

2. Second, by considering the relationships between the components of metabolic syndrome, we may be able to better understand the pathophysiology that links them with each other and with the increased risk of CVD.

3. Third, it facilitates epidemiological and clinical studies of pharmacological, lifestyle and preventive treatment approaches. $(10)$

\section{Risk Factors for Metabolic Syndrome-}

- Stress.

- Sedentary lifestyle.

- Overweight/ Obesity.

- Smoking.

- Diet.

- Aging.

- Hypertension.

- Diabetes mellitus.

- Coronary heart disease.

- Lipodystrophy.

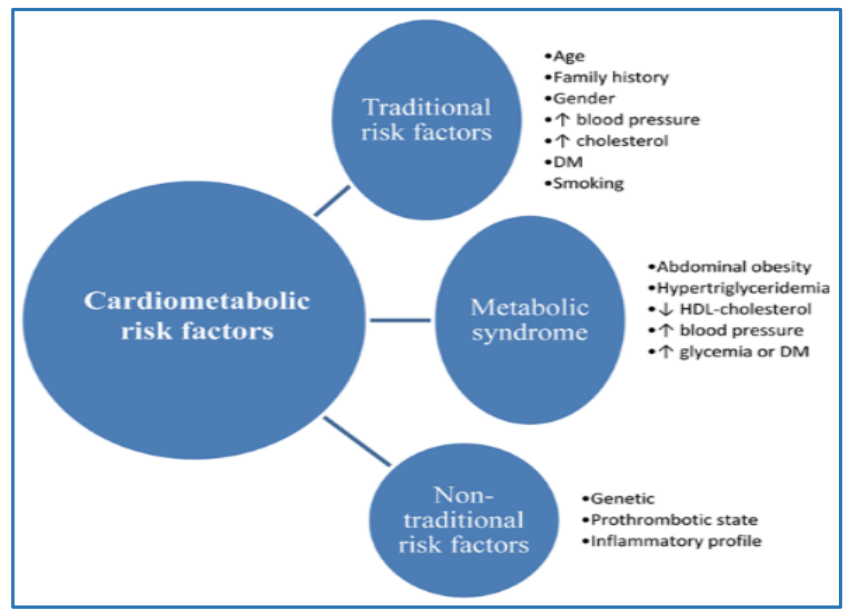

Worldwide prevalence of MetS ranges from $<10 \%$ to as much as $84 \%$ depending on the region, urban or rural environment, composition (sex, age, race and ethnicity) of the population studied and the definition of the syndrome used.(11,12) The prevalence of obesity and the metabolic syndrome is rapidly increasing in India and other south Asian countries leading to increased morbidity and mortality due to type 2 diabetes mellitus (T2DM) and cardiovascular disease (CVD).(13)

\section{Objective}

In the present study, we are attempting to identify the correlation between culturally acceptable anthropometric measure such as neck circumference and fasting lipid profile.
This may help identify the candidates who need more detailed evaluation for metabolic syndrome and cardiovascular evaluation.

\section{MATERIALS AND METHODS}

The study was conducted on patients attending the outpatient department (OPD) for general health check-up at Yenepoya Medical College Hospital. The study was crosssectional study conducted in 201 participants. Informed written consent was obtained from cases for participation in the study and for conducting investigations. The study was conducted between the period of January 2016 and September 2017.

\section{Data Collection}

Patients included in this study were all males and females above 20 years of age and cases with Thyroid disorders. Any neck surgery and pregnant women were excluded. Along with fasting lipid profile, neck circumference was measured in all the participants and parameters evaluated.

\section{Statistical Analysis}

Discrete data was expressed in frequencies and percentages and continuous data was expressed in mean \pm SD [Range]. Pearson's correlation was used to correlate neck circumference with cholesterol and LDL. Data was analysed using the software SPSS version 22.0. $\mathrm{P}<0.05$ was considered significant.

\section{RESULTS}

Total of 201 patients were studied, out of which 145 were males and 56 were females. All patients were above 20 years of age, $32 \%$ patients were between 41 to 50 years and $21 \%$ were between 51 and 60 years of age. Out of 201 patients in this study, 96 patients had Diabetes mellitus, 73 patients had Hypertension and 45 patients had Ischaemic heart disease.

Out of 145 males mean Neck circumference was $36.22 \mathrm{~cm}$, minimal NC was $31.5 \mathrm{~cm}$ and maximum NC was $41 \mathrm{~cm}$. Out of 56 females mean Neck circumference was $33.9 \mathrm{~cm}$, minimal $\mathrm{NC}$ was $28 \mathrm{~cm}$ and maximum NC was $40 \mathrm{~cm}$. Among males, mean cholesterol was 190 and LDL was 118. Among females, mean cholesterol was 193 and LDL was 124 .

\begin{tabular}{|c|c|c|}
\hline & Female & Male \\
\hline Neck & $33.95 \pm 3.16$ & $36.22 \pm 2.11$ \\
Circumference & {$[28-40]$} & {$[31.5-41]$} \\
\hline \multirow{2}{*}{ Cholesterol } & $193.96 \pm 36.63$ & $190.34 \pm 42.29$ \\
& {$[118-290]$} & {$[91-302]$} \\
\hline \multirow{2}{*}{ LDL } & $124.11 \pm 33.30$ & $118.69 \pm 38.02$ \\
& {$[45-217]$} & {$[35-213]$} \\
\hline
\end{tabular}

Data expressed as Mean \pm SD [Range].

With Pearson correlation coefficient between Neck circumference and cholesterol and LDL, there was a significant positive correlation between Neck Circumference and Cholesterol in male and females with $\mathrm{p}<0.001$ in males and $\mathrm{p}=0.02$ in females and significant positive correlation between Neck circumference and LDL in males with $p<0.001$.

According to this study, patients with high neck circumference are associated with high cholesterol and high LDL level. 


\section{DISCUSSION}

In our study conducted at Yenepoya Medical College and Hospital, Mangalore during the period of January 2016 and September 2017, we hypothesised that Neck Circumference is related to dyslipidaemia in Indian population and people with higher value of NC have higher values of fasting lipid profile.

In our study, we measured Neck Circumference and laboratory values including fasting lipid profile (cholesterol, triglyceride, HDL and LDL). The cut-off values for Neck Circumference in males was $37 \mathrm{~cm}$ and females was $34 \mathrm{~cm}$ and cardiometabolic syndrome diagnosed based on criteria of NCEP ATP III criteria.

\section{Ben Noun et al(14)}

Did a study to show Neck circumference as a simple screening measure for identifying overweight and obesity patients. A total of 979 patients were enrolled which include test sample and validation sample, among them 460 were males and 519 were females. This study indicates that NC was associated with age, weight, waist and hip circumferences, waist: hip ratio and BMI for men and women. Thus, a strong association was found between NC and conventional overweight and obesity indexes. $\mathrm{NC}>37 \mathrm{~cm}$ for men and $>34 \mathrm{~cm}$ for women identified subjects with BMI $>25.0 \mathrm{~kg} / \mathrm{m} 2$ with $98 \%$ to $99 \%$ sensitivity for men and $99 \%$ to $100 \%$ for women, $89 \%$ to $92 \%$ specificity for men and $98 \%$ to $100 \%$ for women. These findings indicate that NC can be used as a simple, easy to perform, quick test that can be used to identify overweight or obese patients. Although, NC shows a strong correlation with both overweight and obesity, it is reasonable to consider it as a screening test. Men with NC $<37 \mathrm{~cm}$ and women with $\mathrm{NC}<34 \mathrm{~cm}$ do not require additional evaluation. Patients above these levels require a more comprehensive evaluation of their overweight or obesity status.

\section{Ben Noun et al}

Once again in 2004 observed relationship between changes in neck circumference and changes in blood pressure. In this longitudinal cohort study, the study group was comprised of 364 subjects (155 men and 209 women) with no known major medical conditions who were not receiving any medication therapy. They found that changes in systolic BP and diastolic BP correlated positively with changes in NC and other components of the metabolic syndrome.

\section{Liubov et al(15)}

Conducted a study to determine a relationship between neck circumference (NC) and risk factors for coronary heart disease by evaluating the components of the metabolic syndrome. A total of 561 subjects (231 males and 330 females) with no major medical conditions and who were not receiving any medications. Study included NC, WC, BMI, BP, FBS and waist-hip ratio. In this study NC was also strongly correlated with SBP, DBP, total cholesterol, LDL-cholesterol, triglycerides, fasting glucose and uric acid levels, NC of $>38$ $\mathrm{cm}$ in males and $>34 \mathrm{~cm}$ in females is correlated positively with factors of metabolic syndrome; therefore, are correlated with the change in risk of cardiovascular disease.

\section{Guang Ran Yang et al (16)}

Investigated the association between neck circumference and central obesity, overweight and metabolic syndrome in Chinese individuals with type 2 diabetes. A total of 3185 diabetic subjects were recruited from 15 community health centres in Beijing using a multistage random sampling approach. ROC analysis showed that the area under the curve of $\mathrm{NC}$ and central obesity was 0.77 for men and 0.75 for women, respectively. $\mathrm{NC}>38 \mathrm{~cm}$ for men and $>35 \mathrm{~cm}$ for women were the best cut-off points for determining subjects with overweight. A NC $>39 \mathrm{~cm}$ in men and $>35 \mathrm{~cm}$ in women was the best cut-off point to determine subjects with metabolic syndrome. In present study, NC is positively related with BMI, waist circumference and metabolic syndrome in Chinese individuals with Type 2 diabetes.

\section{Apurva Sawanth et al(4)}

Done a clinical study of prevalence of Metabolic Syndrome in Urban India. A total of 560 subjects, who attended the free Cardiac evaluation camp were recruited in the study. The study concluded that prevalence of MS was double in males as compared to females. The study revealed the increased prevalence of MS to be more in 41 - 60 years, suggesting that this group is at risk of developing CAD. It was also found that high percentage prevalence of overweight and obesity was one of the major driving forces in the development of MS. Therefore, early identification of the metabolic abnormalities and appropriate intervention may be of primary importance in populations especially having high prevalence.

\section{Giovanni et al(17)}

Investigated to show Neck Circumference as a Predictor of Metabolic Syndrome and Obstructive Sleep Apnoea in ShortSleeping Obese Men and Women. It was a cross-sectional study of obese men and premenopausal obese women sleeping less than $6.5 \mathrm{hrs}$. per night. They enrolled 120 individuals ( 92 women), aged $40.5 \pm 6.9$ years and body mass index (BMI) of $38.6 \pm 6.5 \mathrm{~kg} / \mathrm{m}^{2}$. Metabolic syndrome was found in $41 \%$ and OSAS in $58 \%$ (28\% had both). A NC of $\geq 38 \mathrm{~cm}$ had a sensitivity of $54 \%$ and $58 \%$ and a specificity of $70 \%$ and $79 \%$ in predicting the presence of metabolic syndrome and OSAS, respectively. According to this study, greater NC is associated with OSAS and metabolic syndrome in short-sleeping obese men and premenopausal obese women.

In our study, we have identified that there is a significant positive correlation between Neck Circumference and Cholesterol in males and females, $p$ value of 0.00 in males and $\mathrm{p}$ value of 0.02 in females and significant positive correlation between Neck circumference and LDL in males with $\mathrm{p}$ value of 0.00 .

According to this study, patients with high neck circumference are associated with high cholesterol and high LDL level.

\section{CONCLUSION}

According to this study, patients with high neck circumference are associated with high cholesterol and high LDL level. NC may be used as a simple and time saving screening measure to identify cardiometabolic risk factors like dyslipidaemia in patients. This helps in conserving time and resources for patients who are in need of the same in our 
present setting. Not many studies are available on this on Indian population and hence further large scale studies would justify and validate the findings of our current study.

\section{REFERENCES}

[1] Grunfeld C, Rimland D, Gibert CL, et al. Association of upper trunk and visceral adipose tissue volume with insulin resistance in control and HIV-infected subjects in the FRAM study. J Acquir Immune Defic Syndr 2007;46(3):283-90.

[2] Onat A, Hergenç G, Yüksel H, et al. Neck circumference as a measure of central obesity: associations with metabolic syndrome and obstructive sleep apnea syndrome beyond waist circumference. Clin Nutr 2009;28(1):46-51.

[3] Zhou J, Ge H, Zhu M, et al. Neck circumference as an independent predictive contributor to cardiometabolic syndrome. Cardiovasc Diabetol 2013;12:76.

[4] Sawant A, Mankeshwar R, Shah S, et al. Prevalence of metabolic syndrome in Urban India. Cholesterol 2011;2011:1-7.

[5] Kaur J. A comprehensive review on metabolic syndrome. Cardiol Res Pract 2014;2014:943162.

[6] Alberti KG, Zimmet P, Shaw J. Metabolic syndrome--a new world-wide definition. A consensus statement from the international diabetes federation. Diabet Med 2006;23(5):469-80.

[7] Sookoian S, Pirola CJ. Genetics of the cardiometabolic syndrome: new insights and therapeutic implications. Ther Adv Cardiovasc Dis 2007;1(1):37-47.

[8] Yuan G, Hegele RA. Genetic forms of the cardiometabolic syndrome: what can they tell the clinician? J Cardiometab Syndr 2007;2(1):45-8.
[9] Jensen MD. Role of body fat distribution and the metabolic complications of obesity. J Clin Endocrinol Metab 2008;93(11 Suppl 1):S57-63.

[10] Beilby J. Definition of metabolic syndrome: report of the national heart, lung, and blood Institute/American heart association conference on scientific issues related to definition. Clin Biochem Rev 2004;25(3):195-8.

[11] Kolovou GD, Anagnostopoulou KK, Salpea KD, et al. The prevalence of metabolic syndrome in various populations. Am J Med Sci 2007;333(6):362-71.

[12] Desroches S, Lamarche B. The evolving definitions and increasing prevalence of the metabolic syndrome. Appl Physiol Nutr Metab 2007;32(1):23-32.

[13] Misra A, Khurana L. The metabolic syndrome in South Asians: epidemiology, determinants, and prevention. Metab Syndr Relat Disord 2009;7(6):497-514.

[14] Ben-Noun L, Sohar E, Laor A. Neck circumference as a simple screening measure for identifying overweight and obese patients. Obes Res 2001;9(8):470-7.

[15] Ben-Noun L, Laor A. Relationship of neck circumference to cardiovascular risk factors. Obes Res 2003;11(2):226-31.

[16] Yang GR, Yuan SY, Fu HJ, et al. Neck circumference positively related with central obesity, overweight, and metabolic syndrome in Chinese subjects with type 2 diabetes: Beijing community diabetes study 4. Diabetes Care 2010;33(11):2465-7.

[17] Cizza G, de Jonge L, Piaggi P, et al. Neck circumference is a predictor of metabolic syndrome and obstructive sleep apnea in short-sleeping obese men and women. Metab Syndr Relat Disord 2014;12(4):231-41. 